\title{
Mechanisms and empirical equations for deformation and some principles of alloy design
}

\author{
T BALAKRISHNA BHAT \\ Defence Metallurgical Research Laboratory, Kanchanbagh, Hyderabad 500258, India
}

\begin{abstract}
Some aspects of the deformation behaviour of solids at very high, moderately high and low strain rates are discussed. In the very high strain rate region, deformation equations and the physics of the shock front are analysed to propose a route to lower energy dissipation at the shock front. In the moderately high strain rate region, alloy design principles for maximizing the deformation resistance are outlined. In the low strain rate region, an analysis of the physical basis for the power law creep equation is presented. Some physical arguments are presented as a rationale for the high stress exponents and activation energies often observed in particle-strengthened alloys. The additivity of strain rates by various mechanisms is also briefly discussed.
\end{abstract}

Keywords. Deformation mechanisms; empirical equations; dislocation dynamics; shock front; impact resistance; alloy design.

\section{Introduction}

The behaviour of solids under deformation depends on the operating stress level, the temperature, and many material properties such as the elastic modulus, the microstructure and the properties of the individual constituents. The behaviour is indeed complex and can be described only by semiquantitative and quasi-empirical relations. In the very high strain rate region where shock waves predominate in the flow process, the microstructure and initial temperature are relatively unimportant. The crystalline state and basic material properties such as the modulus and density are important in this region. In the lower strain rate region such as that observed in the ballistic testing of materials, the stress-strain diagrams, the glide resistances, the phonon drag and the temperature play important roles. In the low strain rate region as in creep, the operating temperature and stress are most important. A smaller but significant role is also played by the microstructure. The regimes of deformation rates and stresses of interest in the present discussion are shown in figure 1.

\section{Deformation at very high pressures and strain rate}

Pressure impulses injected into materials generate shock waves. At low pressures the shock front consists of purely elastic strains and the wave propagates over large distances with relatively little attenuation. A typical example of such a wave is that of sound waves generated by gentle knocks on any solid. More intense waves such as those generated during hollow charge collisions or in implosions intended for thermonuclear fusion generate high nonelastic strains at the wave-fronts. Such waves suffer large drags and tend to get dissipated. This drag is in some cases undesirable, for example in 


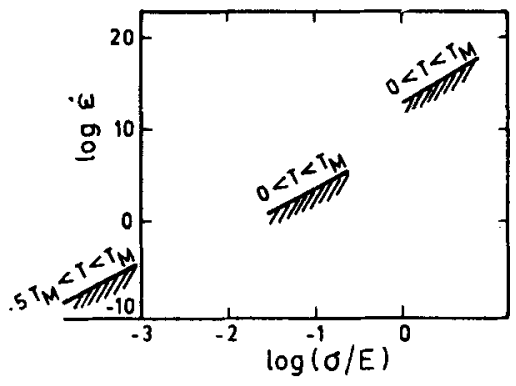

Figure 1. Stress-strain rate regimes considered in this paper.
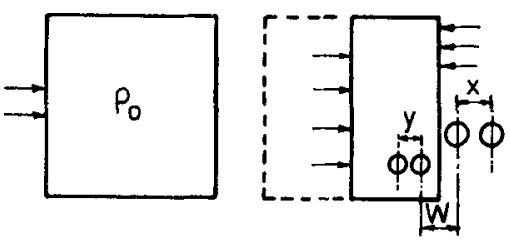

Figure 2. Schematic diagram illustrating the strain at a shock front: $\varepsilon=(x-y) / x$.

thermonuclear experiments. We may therefore analyse the drag mechanisms in terms of a specific question, namely, how to reduce the drag.

At the shock front a low density phase is transformed to a high density phase. This transition induces both bulk and shear strains at the front (Gilman 1979). Figure 2 illustrates the situation at the shock front. The strain there may be written as

$$
\varepsilon=\left(\rho-\rho_{0}\right) / \rho_{0},
$$

where $\rho_{0}$ is the initial density and $\rho$ is the shocked density. The power dissipated at the moving front is

$$
G=B \dot{\varepsilon}=B v_{\text {sh }} / W,
$$

where $B$ is the drag coefficient, $v_{\mathrm{sh}}$ is the speed of the shock front, and $W$ is the width of the transition region between the shocked and the unshocked state. The power dissipated at the shock front is thus controlled by the parameters $B$ and $W$.

In crystalline solids the shock front moves via the coordinated motion of atoms. The interface between the shocked and the unshocked regions is thought to consist of a set of interface dislocations and a compressed zone (figure 3). The areal density of dislocations, $\rho_{\text {dis }}$, depends on the compression ratio according to

$$
\rho_{\mathrm{dis}}=\frac{1}{\sqrt{2} b}\left[1-\left(\rho_{0} / \rho\right)^{1 / 3}\right],
$$

where $b$ is the Burgers vector. Figure 4 is a plot of this dependence. The dislocation spacing approaches $b$ at high compression ratios. The shear strain rate at those dislocations (which travel with the shock front) becomes $\dot{\varepsilon}=v_{\text {sh }} / b$. Typical values are $v_{\mathrm{sh}} \simeq 10^{7} \mathrm{~cm} / \mathrm{sec}$ and $b=2.5 \times 10^{-8} \mathrm{~cm}$, so that $\dot{\varepsilon} \simeq 4 \times 10^{14} \mathrm{sec}^{-1}$. At such a high strain rate, even in a material as fluid as water (viscosity $\sim 10^{-2}$ poise) the drag becomes $4 \times 10^{4} \mathrm{~kg} / \mathrm{mm}^{2}$ ! Shock pulses therefore attenuate extremely rapidly. One way to reduce this drag pressure would be the use of metallic glasses which have a liquid-like structure on either side of the shock front. The intense localisation of strains arising from the smallness of the Burgers vector of the dislocations is then avoided.

\section{Deformation at moderately high strain rates}

At lower stress levels (the middle shaded region in figure 1), the deformation front advances at subsonic speeds. A typical example in this range of strain rates is the 


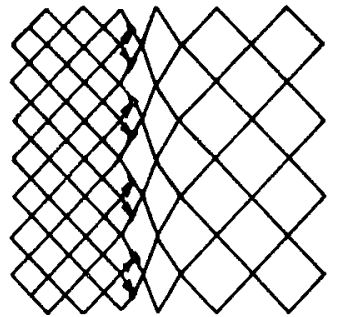

Figure 3. Schematic illustration of the interface structure at shock fronts in crystalline materials.

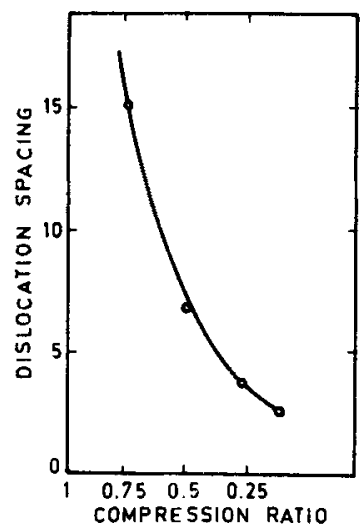

Figure 4. Variation of dislocation spacing (in units of $b$ ) at the shock front as a function of the compression ratio.

deformation armour under ballistic attack. Strain in this regime normally occurs by dislocation movement. The flow is governed by high-velocity dislocation dynamics. The equation of motion for a dislocation line is of the form (Kocks et al 1973)

$$
M \ddot{y}+B \dot{y}=b(\sigma-\tau),
$$

where $M$ is the 'mass' of the dislocation, $B$ is the viscous drag coefficient, $\sigma$ is the applied stress, and $\tau$ is the lineglide resistance of the dislocation element against slow glide. Clearly, $M, B$ and $\tau$ must be maximised for increasing the strength of the material. The 'mass' $M$ of the dislocation can be shown to be

$$
M=U / v_{s}^{2}=1 / 2 b^{2} \rho,
$$

where $U$ is the line energy per unit length of the dislocation, $v_{s}$ is the velocity of sound, and $\rho$ is the material density of the material. Hence high density materials (e.g., steel) and materials with large Burgers vectors (e.g., intermetallics) offer greater resistance to dynamic flow. The drag coefficient $B$ is controlled by the Peierls potential and the phonon-dislocation interaction. Dissipation of energy occurs when dislocations interact with phonons. In addition, shear-stress fluctuations due to phonons cause dislocations to flutter. Nonlinear elastic strains near dislocations also scatter phonons because of local changes of modulus and density (and hence of the speed of elastic waves). These mechanisms retard moving dislocations. Further, a moving dislocation has to build up and relax elastic strains, and this process is also retarded by the interaction with phonons. All these factors lead to a drag coefficient of the form (Kocks et al 1973)

$$
B=k_{B} T / \Omega \omega,
$$

where $\Omega$ is the atomic volume and $\omega$ is the atomic frequency. In contrast to the other mechanisms resisting flow, the $\operatorname{drag} B$ increases with temperature. Moreover, there is also a rapid increase of $B$ when the speed of the dislocation $\gtrsim 0.5 v_{s}$. This is because the strain field of the dislocation begins to be compressed into smaller volumes (for lack of time!) at any given location. However, the discrete nature of the atoms and the low compressibility of atomic cores puts an upper limit to this contraction and allows 
'supersonic' speeds to be reached at high stress levels, as in the shock waves discussed earlier.

Turning to the parameter $\tau$ (the line glide resistance) in (4), we observe first that this resistance normally varies with the location on the glide plane. The Peierls resistance, for instance, varies periodically with a wavelength $b$. Solute atoms provide small discrete obstacles. Precipitates, interphase interfaces and cell or grain boundaries provide a coarser but stronger set of obstacles. Some of these obstacles are repulsive to dislocations, some are attractive and some are energy-storing. A schematic glideresistance diagram is shown in figure 5. The spatial variation of the effective driving force on the dislocation results in an oscillatory motion superposed on its steady state motion. This induces radiation of elastic energy from the moving dislocation into the crystal. As this energy must also be supplied by the applied stress, the hills and valleys in the glide-resistance diagram contribute to the drag. The varying effective driving force also causes rapid acceleration or deceleration of the dislocation as it moves in the glide plane. The dislocation may then be able to overcome an obstacle on its path at least in part through its inertia. Attractive obstacles are specially prone to this phenomenon, for the energy picked up in the 'downhill' motion of the dislocation can facilitate its subsequent travel 'uphill'. Similarly, many small obstacles met with after passage through a large repulsive obstacle can be overcome dynamically. It is also possible for a moving dislocation to interact with near-by dislocations and transfer energy to them. Thus, under dynamic conditions obstacles incorporated in the matrix begin to lose their individuality and efficacy and the macroscopic concepts of flow stress may no longer be valid.

The onset of this dynamic behaviour is influenced by many factors. Higher values of the viscosity and larger obstacles sizes or spacing postpone the onset to higher dislocation velocities. Larger values of $M$ and $b$ favour an early transition. For obstacles of width smaller than a few Burgers vectors, dynamic effects emerge when (Kocks et al 1973)

$$
(\sigma / G)>\left(k_{B} T / \Omega G\right)^{2},
$$

where $\sigma$ is the stress level and $G$ is the shear modulus. A rationale for specifying low- $T$ impact properties for armour materials is hidden in this above equation, because low temperatures bring out the dynamic effects at lower stresses and strain rates. This also suggests that the results of low- $T$ tensile tests may correlate better with ballistic performance when the highest suitable test temperature is chosen by the onset of simultaneous decrease in strength and ductility.

Certain alloy design criteria (for good ballistic performance) also emerge from this discussion. A material that must resist flow at the strain rates, presently under

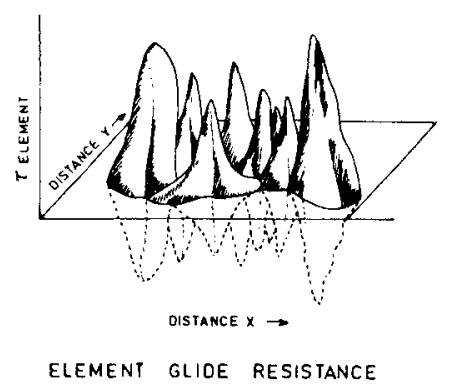

Figure 5. Schematic glide-resistance diagram in multiphase materials. 
consideration, should not exhibit significant dynamic weakening. Thus, a high Peierls stress is particularly detrimental. Also undesirable are microstructures with precipitates that almost touch each other, as are attractive precipitates, even when they are strong. Long range barriers such as subgrains, martensite lath boundaries, strong and large ( $>2000 \AA$ ) repulsive precipitates, or repulsive-type solute elements would be the ideal candidates for the purpose at hand.

\section{Deformation at low stresses and strain rate}

Even at low stresses materials can deform provided the temperature is sufficiently high to permit thermal activation of certain microscopic processes. This deformation mode, called creep, has been extensively studied over the years and a variety of equations, mechanisms and explanations have been proposed by various workers.

One particular widely-used semi-empirical equation is the 'power law', according to which the creep rate $\dot{\varepsilon}$ can be expressed as the product of a (small) power of the stress and an exponential function of $T$ :

$$
\dot{\varepsilon}=A(G b / K T)(\sigma / G)^{n} D_{0} \exp (-Q / R T) .
$$

Here $A$ is a constant, $D_{0}$ is the preexponential in the diffusion coefficient, $R$ is the gas constant and $Q$ is the activation energy for diffusion. This equation has been shown to work for simple metals and ionic solids (Bird et al 1969). Nevertheless, many difficulties and unexplained modes of behaviour persist. The magnitude of the creep rate predicted by (8) is often incorrect (Evans and Knowles 1981). The index $n$ lies anywhere between 1 and 6 and sometimes can be as high as 40 (Benjamin and Cairns 1971). The observed activation energy for creep often deviates significantly from that for diffusion, and in addition varies with $T$ and $\sigma$ (Poirier 1978). Moreover, even if there is a correlation between the activation volumes for self-diffusion and creep, one cannot unequivocally conclude that the creep is diffusion-controlled, because it can be shown that any process that depends on the pressure through the modulus will also have the same activation volume (Beyeler 1969). In spite of the large literature extant on the subject, a generallyagreed upon theory is lacking as yet.

\subsection{The stress exponents and the creep deformation mechanisms}

The index $n$ is unity for viscous flow in solids, as in fluids. This is a characteristic of microscopically homogeneous or structureless flow. Diffusional flow as in polycrystalline materials at elevated temperatures also exhibits a linear stress dependence. Basically, the applied stress serves only to generate higher point defect concentrations on grain boundaries under tensile stress relative to boundaries under no stress. The concentrations are exponential functions of $\left(\sigma \Omega / k_{B} T\right)$ which approximate to linear functions of $\sigma$ at low stress levels (Nabarro 1948; Coble 1963).

Several anomalies exist in the dislocation creep region. Stocker and Ashby (1973) have used a heuristic statistical analysis to prove that $n=3$ is the most probable value for dislocation creep. A simple way to see this is as follows: The dislocation density $\propto \sigma^{2}$, while the dislocation velocity $\propto \sigma$, in a viscous flow picture. Orowan's equation then directly leads to $\dot{\varepsilon} \propto \sigma^{3}$. The same conclusion can also be roached if we treat the creep as being controlled by the coarsening of dislocation networks (Evans and Knowles 1981). 
Dislocation creep that is controlled by recovery processes generally has a larger stress exponent. One such process is the mutual annihilation of dislocations with opposite Burgers vectors (or their segments) when they meet each other. If $\rho_{+}$and $\rho_{-}$denote the densities of dislocations with Burgers vectors of opposite signs, the annihilation can be considered as a second order reaction with a rate given by $\dot{\rho}=K_{1} v \rho_{+} \rho_{-}$where $\rho$ is the total dislocation density and $K_{1}$ is a reaction constant. The rate of reaction, and hence of recovery, is evidently greater the higher the dislocation velocity. The strain rate associated with the recovery can be written as $\dot{\varepsilon}=\dot{\rho} b s$ where $s$ is the average distance travelled by a dislocation before annihilation. Substituting a quadratic dependence of the dislocation density on $\sigma$ and a linear dependence of $v$ on $\sigma$, we get

$$
\dot{\varepsilon} \sim \sigma^{4} \text {. }
$$

This recovery strain rate leads to a proportional but larger glide strain rate, which is the observed strain rate. Thus, recovery-controlled creep is expected to have a stress exponent equal to four.

One may be tempted to combine such power laws into a general functional form

$$
\dot{\varepsilon}=\sum_{n} a_{n}(\sigma / G)^{n},
$$

where the $a_{n}$ are independent of $\sigma$, a particular mechanism being associated with each distinct $n$ value. Equation (10) implies that the control of the creep rate shifts to the mechanisms corresponding to higher $n$ values with increasing stress. Often, however, this is not the case. One plausible conclusion from this is that the index $n$ has no specific connection with any mechanism. Alternatively, one may invoke a back stress concept, such that the effective stress for creep is given by

$$
\sigma_{\mathrm{eff}}=\sigma-\sigma_{b}=\sigma-f_{n}(\sigma)
$$

where $\sigma$ is the applied stress and the function $f_{n}$ depends on the specific mechanism concerned. The back stress can be considered as a measure of the energy spent on athermal steps that are essential for creep but do not directly lead to any significant creep strain. A specific illustration of the use of back stress concept is in the creep behaviour of particle-strengthened systems. Unusually large $n$ values are often observed in both diffusional creep and dislocation creep regions (Benjamin and Cairns 1971). Recently, we have shown how the anomalous stress exponents in TD Nichrome and Mar M-200 superalloys fall in line with that for nickel once the back stress arising from strong dislocation-particle interactions is taken into account (Balakrishna Bhat and Arunachalam 1980; Balakrishna Bhat 1981). When the particles are attractive, the back stress appears as a threshold stress; when they are repulsive, the back stress increases with applied stress, reaching a limiting value as shown in figure 6 . The back stress for diffusion creep is believed to be associated with the interaction of grain boundary dislocations with particles at the grain boundaries. It must be mentioned that the term 'back stress' has also been used in several different contexts. For example, it has been identified with the internal stress which includes the average stress field of the dislocation structure together with the cell boundaries (Pahutova and Cadek 1973; Argon et al 1981). In reality, the power law discussed earlier is the result of the interaction of defects among themselves and with the background stress field; the average stress field of the other defects is therefore already taken into account, and it is incorrect to include it once again in the back stress. 


\subsection{Activation energy for deformation}

4.2a Activation energy and the bulk modulus: The activation energy can be considered as the work a solid under load has to perform on itself in temporarily expanding against its own bulk modulus to accommodate the moving unit of flow. Figure 7 is a plot of $Q /\left[\Omega\left(1-P_{f}\right)\right]$ vs the bulk modulus for various metals. $\left(P_{f}\right.$ is the packing factor for the crystal). The approximately linear fit is noteworthy. It is interesting to note also that the activation energy is the energy that gets stored in the empty space $\Omega\left(1-P_{f}\right)$ as the solid momentarily dilates or contracts!

4.2b Activation energy for dislocation creep and the creep mechanism: A power law for creep presupposes that the activation energy for creep is equal to that for diffusion. However, the observed value of the activation energy is not constant. It increases with $T$

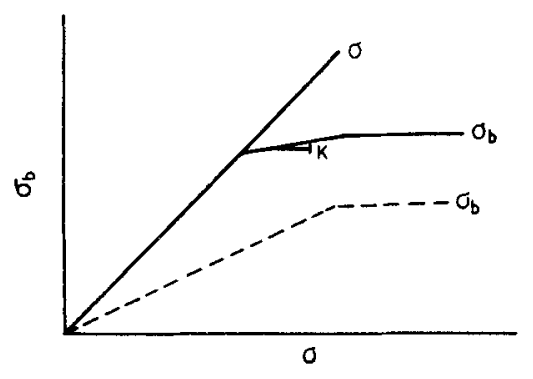

Figure 6. Schematic variation of back stress $\sigma_{b}$ with applied stress $\sigma$ in particlestrengthened alloy systems.

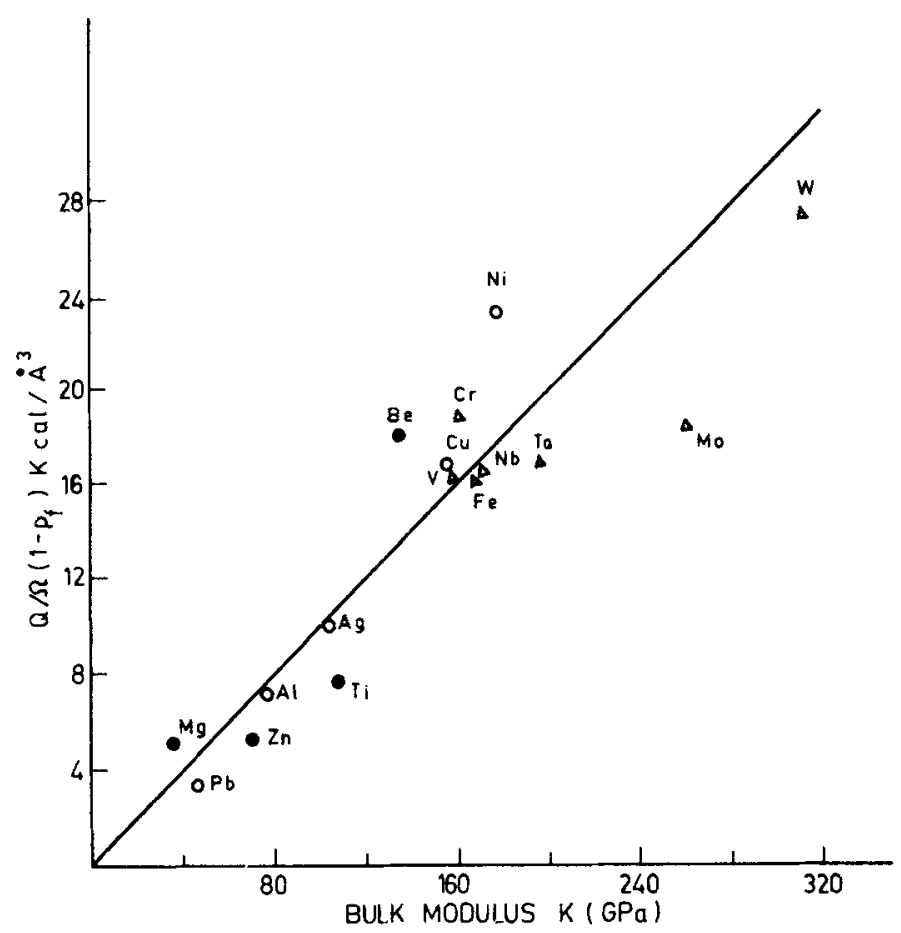

Figure 7. Dependence of $Q /\left[\Omega\left(1-P_{f}\right)\right]$ on bulk modulus for metals. 
to match the activation energy for diffusion only in some small range of homologous temperature which, moreover, is different for each material (Poirier 1978). In particlestrengthened systems anomalously high and variable activation energies have often been reported.

It has often been suggested that the variation of the elastic modulus with temperature should account for the anomaly (Shewfelt and Brown 1974). However, this is not very convincing. Consider a force-displacement diagram between the atoms in a crystal as shown in figure 8. The elastic modulus reflects the rate of energy storage for infinitesimal displacements from the equilibrium value whereas the activation energy reflects the energy needed for a displacement equal to a lattice parameter which is more like the area under the curve. The inadequacy of the effect of $T$ on $E$ as a measure of its effect on $Q$ is obvious.

The apparent activation energy for creep in particle-strengthened systems also depends on the back stress and its variation with temperature. This contribution to the apparent activation energy is given by (Balakrishna Bhat 1981)

$$
\Delta Q=\left[n R T^{2} /\left(\sigma-\sigma_{b}\right)\right]\left[\partial \sigma_{b} / \partial T\right]
$$

For diffusion creep with low $n$ and $\sigma_{b}$, the contribution is relatively small. For dislocation creep with large $n$ and $\sigma_{b}$ (as in TD nichrome), the contribution can be large. Further, $\sigma_{b}$ varies an order of magnitude more rapidly with $T$ than is predicted by the variation of the elastic modulus alone. The reason for this difference is not clear at the moment. Perhaps, with increasing $T$ dislocation climb increases exponentially, facilitating the selection of easier flow paths by a sampling of the field of glide resistance along all three axes. The effective glide resistance (which one may call the 'volume glide resistance') or the back stress for creep will be different from the 'plane glide resistance' derived for a planar distribution of obstacles and a largely in-plane overcoming of obstacles by the dislocations. During creep, dislocations accumulate in difficult-to-glide locations and direct the other dislocations to climb over to easier regions of flow. When once climb has become possible, the back stress changes only slowly with temperature (figure 9).

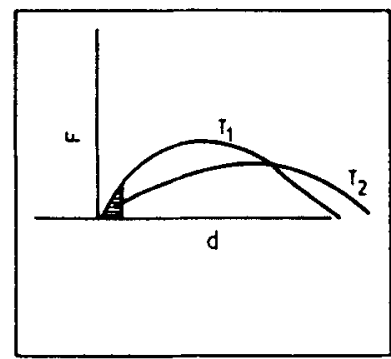

Figure 8. Atomic force-displacement diagram for two different temperatures.

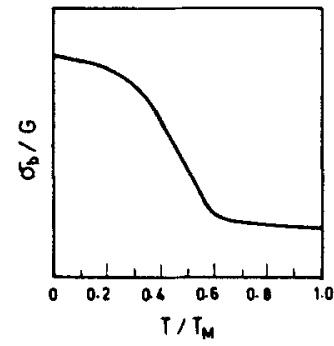

Figure 9. Temperature dependence of the back stress in particle-strengthened alloys. 
A consequence of the volume-sampling hypothesis is that high $n$ and high $Q$ are not necessarily coupled. Even at high temperatures, $\sigma_{b}$ in oDs alloys can be relatively large, giving a large effective $n$. The volume-sampling process reaches saturation above a particular temperature and the back stress then drops slowly with further increase of $T$. The apparent activation energy would then be small in spite of a large $n$ value. Indeed, the results of Benjamin and Cairns (1971) on oDs alloys show a modulus-compensated activation energy close to that for self-diffusion in spite of a high apparent stress exponent of 32.8 at $T=1350^{\circ} \mathrm{C}$. At a lower temperature, a lower $n$ value is obtained, but $Q$ is still twice the activation energy for self-diffusion. Another consequence of volumesampling is that there will be no influence of particle shape on the $T$-dependence of $\sigma_{b}$ for a random distribution of obstacles, since the $T$-dependence arises primarily from the climb-controlled volume-sampling of weaker regions from the same random distribution of obstacles, and not from climb around individual particles. This observation is substantiated by the experiments of Shewfelt and Brown (1974). It would be of interest to perform a computer simulation of this volume-sampling glide process during the creep of particle-strengthened systems, in order to optimize the particle size and distribution for effective resistance to creep flow.

\section{Additivity of creep rates}

An unsolved problem in creep relates to the additivity of creep rates through various mechanisms. The expansion of (10) presupposes such additivity. All theoretical treatments to date have also made this assumption. However, the basic physical processes involved cast a doubt on the validity of this assumption.

Consider for instance diffusion creep and grain boundary sliding. Diffusion creep elongates the grains; when added over all the grains, this leads to directional elongation. However, grain sliding events as in superplastic flow introduce new directions for diffusional creep, with the net result of eliminating diffusion creep on the average.

Then again, dislocation creep pre-empts diffusion creep by consuming vacancies en route as they move from one grain boundary to the other. Dislocation creep also generates ledges on grain boundaries and hinders both diffusion creep and grain boundary sliding. On the other hand, diffusion creep tends to retard the onset of grain boundary sliding by generating nonequiaxed grains. It also retards dislocation creep by not allowing large stress concentrations to build up at the grain boundaries. Thus, each mechanism appears to possess an intrinsic inhibiting effect on the others, which may explain why one obtains well-delineated deformation mechanisms in creep experiments. The opposite can also happen in some cases. For example, dislocation glide and climb favour each other. Dislocation activity at the grain boundary zone can enhance grain boundary diffusivity and hence superplastic strain rates. Computer simulation of the deformation process should be of help in understanding these coupled processes.

\section{Summary}

Our discussion above may be summarized in the form of the following specific questions:

(a) Will metallic glasses exhibit low damping of shock waves? (b) Can low temperature tensile and compressive tests be used to design alloys for good ballistic performance? 
(c) Will attractive precipitates prove inferior to repulsive precipitates in the context of ballistic resistance? (d) Can the Arrhenius term in the creep rate be rewritten as a combination of the bulk modulus, the atomic volume and the packing factor? (e) Is the back stress concept relevant, and is it correct to subtract internal stresses due to defects which move to cause strain? (f) Can a microstructure suitable for optimal creep resistance be designed using the concept of volume glide resistance? (g) Can the strain rates arising from different mechanisms be considered to be additive, even though some of them appear to be mutually inhibiting?

\section{Acknowledgements}

The author acknowledges many useful and inspiring discussions with $\operatorname{Dr} \mathrm{V}$ S Arunachalam and Prof. K Srinivasa Raghavan during the course of this work. He is also grateful to Dr P Rama Rao for inspiring him to write this article. The curves in figure 9 are a result of discussions with Shri A K Gogia.

\section{References}

Argon A S, Print F and Moffatt W C 1981 in Creep and fracture of engineering materials and structures Proc. Int. Conf., Swansea, U.K.

Balakrishna Bhat T and Arunachalam V S $1980 \mathrm{~J}$. Mat. Sci. 151614

Balakrishna Bhat T 1981 Effect of second phase on microstructural development and engineering properties Ph.D. Thesis, Indian Institute of Technology, Madras

Balakrishna Bhat T 1983 Principles for protection Trans. I.I.M. (to be published)

Benjamin J S and Cairns R L 1971 in Modern developments in powder metallurgy (ed) H Hausner (New York: Plenum) 547

Beyeler M 1969 Report CEAR 3715

Bird J E, Mukherjee A K and Dorn J E 1969 in Quantitative relations between properties and microstructure (eds) D G Brandon and A Rosen, (Jerusalem: Israel University Press) p 255

Coble R L 1963 J. Appl. Phys. 341679

Evans H E and Knowles G 1981 in Creep and fracture of engineering materials and structure Proc. Int. Conf. at Swansea, U.K. (eds) B Wilshire and D R Owen J. p 169

Gilman J J 1979 J. App. Phys. 504059

Kocks U F, Argon A S and Ashby M F 1973 Thermodynamics and kinetics of slip (Illinois: Argonne National Laboratory)

Malu M and Tien J K 1975 Ser. Met. 91117

Nabarro F R N 1948 Report of a Conference on the strength of solids 1963 The Physical Society, London 1.

Pahutova M and Cadek 1973 J. Mater. Sci. Eng. 11151

Poirier J P 1978 Acta Metall. 26629

Shewfelt A S W and Brown L M 1974 Philos. Mag. 301125

Stocker R L and Ashby M F 1973 Scr. Met. 7115

\section{Discussion}

K A Padmanabhan: Your statement that dislocations could move as fast as the shock front does not appear to tally with the detailed analysis of J D Eshelby on dynamical effects on dislocations. Moreover, as in special relativity, the mass of a dislocation increases with its velocity. Incidentally, Dr Chidambaram's observation that the density of dislocations at the shock wave front is $\sim 10^{11} \mathrm{~cm}^{2}$ also indicates that the total strain arises from a larger number of dislocations rather than a few moving at extremely high speeds. 
N Kumar: In his book, Frank describes what led him to the theory of the Frank-Read mechanism: the realization that the velocity of a dislocation could never exceed the velocity of sound.

Bhat: When the width and amplitude of the incident impulse are large, shock waves travelling at speeds higher than the speed of sound develop. The strain gradients at the front have to be established in the short duration of the passage of the front, so that the strain rate becomes high. This strain rate can be achieved by either or both the terms in the classical equation $\dot{\varepsilon}=\rho b v+\dot{\rho} b s$ where the symbols are as explained in the text. Clearly, movement of the dislocations is required, $v$ being associated with $\rho$ and $s$ with $\dot{\rho}$. If quasistatic dislocations alone account for the shock front, the shocked stress state would acquire permanence in the material. When shock waves can travel faster than the speed of sound, dislocations too can do the same. Physically both share the same important characteristics - strains and strain gradients in their vicinity.

S N Bandhopadhyay: The basic equations of plasticity like those for the velocity field, strain field, localized modes of deformation etc., do not appear to be taken care of properly in the finite element modelling of deformation.

Bhat: I think that the finite element method specialises in taking care of localized modes of deformation without sacrificing the basic equations of plasticity.

K A Padmanabhan: Did you measure the back stress in your experiment by either the stress reduction or stress relaxation method? Otherwise you are just introducing an adjustable variable.

Bhat: The existence of the back stress has not been experimentally established, and it cannot be measured directly. It is felt only by the dislocation that is moving in a structure. Even though the back stress is an adjustable empirical variable it has a certain consistency associated with it. When we introduce this parameter in order to bring the stress exponents to normal values, the anomalous activation energies in such materials automatically come down to normal values for diffusion. This certainly suggests that back stress is a parameter that has some fundamental significance associated with it.

V Balakrishnan: Regarding the computer simulation results on deformation, if you begin with empty spaces between grains, should you not expect further cavitation to occur? Or do you have hydrostatic stress, and the cavities just get filled in?

Bhat: We apply hydrostatic or uniaxial compressive loading in order to simulate hot pressing conditions.

V Balakrishnan: Within a single grain or element, what sort of compatibility conditions do you put in to ensure that no holes are formed?

Bhat: Strain compatibility conditions are put in to ensure that any particular mode has a unique displacement. Regarding your observations on the additivity vs interdependency of various creep mechanisms, another interesting example is provided by grain boundary sliding and diffusion creep. Grain boundary sliding occurs via the climb as well as glide of dislocations at the boundary. Climb generates vacancies which can in turn lead to vacancy creep. 